\title{
Efectos de método en el Inventario de Depresión Estado-Rasgo (IDER): un análisis sem
}

\section{method Effects in State-Trail Depression Inventory [ST-DEP]: Al sem finalysis Efeitos de método no Inventário de Depressão Estado-Rasgo (IDER]: uma análise SEm}

\author{
Sergio Dominguez-Lara* \\ César Merino-Soto* \\ Universidad San Martín de Porres, Lima, Perú.
}

Doi: http://dx.doi.org/10.12804/revistas.urosario.edu.co/apl/a.4151

\section{Resumen}

El objetivo del presente estudio instrumental fue determinar si existe un efecto del método asociado a la formulación de ítems invertidos que conforman la subescala eutimia del Inventario de Depresión Estado-Rasgo (IDER). Se contó con una muestra intencional de 357 estudiantes universitarios, de los cuales 147 fueron varones y 210 mujeres, cuyas edades estuvieron entre 16 y 44 años (media: 20,21). Se evaluaron cuatro modelos mediante análisis factorial confirmatorio: un modelo de dos factores correlacionados, eutimia y distimia; un modelo que agrupa todos los ítems en un solo factor, con un factor de método independiente asociado a los ítems del factor eutimia (ítems invertidos); un modelo que evalúa el ajuste de las secciones rasgo y estado simultáneamente, manteniendo el modelo de dos factores oblicuos, lo cual sirvió como línea base para comparar el cuarto modelo, que incluye el factor del método común a las secciones estado y rasgo. Los modelos dos y cuatro presentaron el mejor ajuste entre los cuatro propuestos, aunque sin ser definitivamente mejores que los modelos de factores oblicuos. Ante ello, no se demostró la presencia determinante de un factor de método asociado a los ítems invertidos de la escala eutimia. Fueron discutidas las implicancias de los resultados.

Palabras clave: depresión, efecto del método, análisis factorial confirmatorio, ítems invertidos, evaluación.

\section{fibstract}

The aim of this instrumental study was to determine whether there is a method effect associated to the inverted items of the eutimia subscale of the State-Trait Depression Inventory. It featured an intentional sample

* Sergio Dominguez-Lara, César Merino-Soto, Instituto de Investigación de Psicología, Universidad San Martín de Porres, Lima, Perú.

La correspondencia relativa a este artículo debe ser enviada a Sergio Alexis Dominguez Lara, Instituto de Investigación de Psicología, Universidad de San Martín de Porres, Av. Tomás Marsano 242 (5to piso), Lima 34, Perú. Correos electrónicos: sdominguezl@usmp.pe, sdominguezmpcs@gmail.com

Cómo citar este artículo: Dominguez-Lara, S. y Merino-Soto, C. (2018). Efectos de método en el Inventario de Depresión Estado-Rasgo (IDER): un análisis SEM. Avances en Psicología Latinoamericana, 36(2), 253-267. DOI: http://dx.doi.org/10.12804/ revistas.urosario.edu.co/apl/a.4151 
of 357 college students, 147 men and 210 women, aged between 16 and 44 years (mean: 20.21). Four models were evaluated using confirmatory factor analysis: a model of two correlated factors, eutimia and dysthymia; a model that includes all the items in one factor, an independent method factor associated to the items of eutimia (inverted items); a model that assesses the fit of state and trait sections simultaneously, maintaining the two oblique factors model, which served as a baseline for comparing the fourth model, which includes the common method factor to state-trait sections. The second and fourth model showed the best fit between the four proposed models, but were not definitely better than the models of oblique factors. In response, the determining presence of a method-factor associated to the inverted items of eutimia scale was not demonstrated. The implications of the results were discussed. Keywords: depression, method effect, confirmatory factorial analysis, negatively worded items, assessment.

\section{Resumo}

O objetivo do presente estudo instrumental foi determinar se existe um efeito do método associado à formulação de itens invertidos que conformam a subescala Eutimia do Inventário de Depressão Estado-Rasgo. Teve-se uma amostra intencional de 357 estudantes universitários, dos quais 147 foram homens e 210 mulheres, cujas idades estiveram entre 16 e 44 anos (Média: 20.21). Se avaliaram quatro modelos mediante análise fatorial confirmatório: um modelo de dois fatores correlacionados, Eutimia e Distimia; um modelo que agrupa todos os itens do fator Eutimia (itens invertidos); um modelo que avalia o ajuste das seções Rasgo e Estado simultaneamente, mantendo o modelo de 2 fatores oblíquos, o qual serviu como linha base para comparar o quarto modelo, que inclui o fator do método comum às seções Estado e Rasgo. O segundo e quarto modelo apresentaram o melhor ajuste entre os quatro modelos propostos, ainda que sem ser definitivamente melhores que os modelos de fatores oblíquos. Ante isto, não se demonstrou a presença determinante de um fator de método associado aos itens invertidos da escala Eutimia. Foram discutidas as implicações dos resultados. Palavras-chave: depressão, efeito do método, análise fatorial confirmatório, itens invertidos, avaliação.

\section{Introducción}

Los trastornos depresivos se constituyen como un problema de salud pública a nivel mundial (WHO, 2010), y por las consecuencias negativas que trae para la persona es que la Organización Mundial de la Salud afirma que para el 2020 la depresión será la segunda causa de incapacidad en el mundo (WHO, 2002). En el marco de acciones preventivas o de rehabilitación psicológica, la instrumentación para obtener una medida estandarizada de la depresión es sine qua non para actuar desde un marco científico. Por lo tanto, es necesario contar con instrumentos de evaluación que demuestren las mejores evidencias de validez y confiabilidad que respalden las inferencias sobre la detección temprana de algunos síntomas de depresión. Una de estas medidas, que es el objetivo del presente estudio, es el Inventario de Depresión Estado-Rasgo (IDER) (Spielberger, Agudelo \& Buela-Casal, 2008), el cual presenta ventajas sobre algunos instrumentos conocidos, como la Escala Autoadministrada de Depresión (Zung, 1965) y el Inventario de Depresión de Beck-II (Beck, Steer \& Brown, 2006). Estas ventajas son respecto a sus aspectos psicométricos, su eficiente balance costo-beneficio y la homogeneidad del constructo medido (Sotelo et al., 2012). Otra de las características más representativas del IDER es que permite diferenciar entre la experiencia actual de la persona (estado) y su forma habitual de comportarse (rasgo) en lo que respecta al componente afectivo de la depresión (Spielberger et al., 2008). Esto es de gran valor clínico para diferenciar la experiencia en dos marcos de tiempo y orientarse específicamente hacia una de las áreas constitutivas de la depresión: los trastornos afectivos (Agudelo et al., 2005). 
Esta diferenciación entre la frecuencia de aparición de los síntomas (rasgo) y la afectación que tiene la persona según dicha sintomatología (estado) permite comprender cómo la presencia de rasgos depresivos aumenta la probabilidad de vulnerabilidad a episodios depresivos (Agudelo, 2009; Agudelo, Spielberger \& Buela-Casal, 2007). Del mismo modo, esta separación entre estado y rasgo hace que el instrumento sea más sensible a cambios pequeños en su nivel de afectación, dado el carácter transitorio de estos, y hace más probable la aplicación del instrumento en población no consultante (Agudelo et al., 2005). Además de la versión profesional del IDER (Spielberger et al., 2008), en estudios previos se han resaltado las propiedades psicométricas del instrumento en diversos contextos hispanohablantes, como en España (Agudelo, 2009), Colombia (Agudelo, Gómez \& López, 2014; Ocampo, 2007), Chile (Vera-Villarroel et al., 2008) y Perú (Dominguez, 2011; Merino, Pflucker \& Riaño-Hernández, 2013; Sotelo et al., 2012). Todos estos estudios anteriores convergen en que en cada sección del instrumento (estado y rasgo) hay dos variables latentes empíricamente diferenciadas: eutimia y distimia, tal como fueron diseñadas conceptualmente por Spielberger en sus estudios iniciales de construcción del IDER (Spielberger et al., 2008). La eutimia queda definida como "[...] grado en el que un estado de depresión está presente en el momento de la evaluación en términos de ausencia de afectividad positiva [...]" y la distimia como "[...] grado en el que un estado de depresión está presente en el momento de la evaluación en términos de presencia de afectividad negativa [...]" (Spielberger et al., 2008, p. 12). Dentro de estos hallazgos, la varianza verdadera retenida por los puntajes, estimada desde la teoría clásica de los test, tiende a ser excelente para propósitos descriptivos grupales e individuales.

Por otro lado, cabe mencionar que la subescala eutimia de las dos secciones (estado y rasgo) presenta ítems cuya calificación es invertida a fin de resaltar que a mayor puntaje menor afectividad positiva. No obstante, ninguno de los estudios citados ha mencionado el posible sesgo que pueden introducir los ítems invertidos que integran la subescala eutimia, de la sección tanto de estado como de rasgo en las respuestas al IDER, y que pueden ser representados configuracionalmente como un factor adicional irrelevante a los constructos medidos, producido por el método empleado (la inversión de los ítems). Es decir, no se ha probado empíricamente si la presencia del factor conocido como eutimia se debe más a una diferenciación sustantiva del constructo frente a la distimia o al método de medida utilizado. Este problema es razonable que ocurra en instrumentos de autorreporte que contengan ítems de orientación opuesta al constructo, el cual es conocido como varianza del método (Lance, Dawson, Birkelbach \& Hoffman, 2010; Podakoff, MacKenzie, Lee \& Podsakoff, 2003; Spector, 2006).

La varianza del método, de forma general, hace referencia a un error sistemático o contaminación debido al método de medida y no al rasgo que se pretende evaluar (Campbell \& Fiske, 1959; Krosnick, 1999). Una de las consecuencias que ocasiona es complicar los análisis factoriales de las escalas que se estudian, pues pueden generar factores no sustantivos y complicaciones en la interpretación de la solución factorial en su totalidad (Oliver, Sancho, Galiana, Tomás \& Gutiérrez, 2012). A lo largo del tiempo, el estudio de dicho sesgo incluye aspectos sobre: considerar en un cuestionario tanto preguntas abiertas como cerradas, el etiquetado de los puntos de la escala, control de la deseabilidad social, aquiescencia, entre otros aspectos que parecen ubicuos a los métodos de autoinforme (Krosnick, 1999; Schaeffer $\&$ Presser, 2003). Aunque fue el sesgo de aquiescencia lo que ha generado mayores controversias (Krosnick, 1999), su solución más habitual (el uso de ítems invertidos propuesto) también se ha llevado al debate. En este sentido, el uso de ítems invertidos ha sido recomendado por largo tiempo en diversos textos (por ejemplo, Nunnally, 1978) 
con el fin de controlar el sesgo de aquiescencia (Ferrando \& Lorenzo-Seva, 2010); sin embargo, esta propuesta podría interferir con el análisis de la estructura latente del instrumento que se pretenda investigar (Carmines \& Zeller, 1979). Un ejemplo clásico al respecto es el realizado por Carmines y Zeller (1979), quienes estudiaron la Escala de Autoestima Global (Rosenberg, 1965), la cual presenta dos grupos de ítems: cinco ítems en positivo, cuyo puntaje va en la dirección del constructo evaluado, y cinco ítems invertidos, cuya puntuación va en la dirección opuesta a la del constructo a evaluar. Tras un análisis factorial exploratorio encontraron dos factores, uno conformado por los ítems en positivo y otro por los ítems invertidos, lo que sugería dos constructos interpretables. Sin embargo, los correlatos de ambos factores con 16 criterios externos relevaron que las diferencias entre correlaciones de los dos factores de autoestima con cada uno de los criterios no fueron estadísticamente significativas, es decir, indicaban que los dos factores no hacían referencia a constructos sustantivamente diferentes (Tomás, Galiana, Hontangas, Oliver \& Sancho, 2013). Las investigaciones que abordaron el sesgo en esta escala de autoestima concluyeron que se trataba de un factor adicional que influenciaba el puntaje latente de autoestima y se identificaba como el efecto del método de medida relacionada a la inversión de los ítems (Oliver et al., 2012; Tomás et al., 2013).

Si bien es cierto que los trabajos germinales en este campo fueron realizados bajo un enfoque multivariado exploratorio (análisis factorial exploratorio) o con correlaciones bivariadas (por ejemplo, Carmines \& Zeller, 1979), dichos procedimientos no presentan garantías al momento de comparar estructuras factoriales ni de distinguir el error de medición en las correlaciones realizadas (Marsh, 1996). En contraste, los métodos sustentados en la metodología de las ecuaciones estructurales (structural equations modeling [SEM]) son más adecuados (Tomás \& Oliver, 1999), específicamente en los análisis del modelo de medición (por ejemplo, confirmatory factor analysis [CFA]). Dentro de este marco, existen diversos métodos estadísticos para controlar el sesgo del método desde un enfoque confirmatorio (CFA) con rasgos y unicidades correlacionadas (CFA-CTCU) y CFA con rasgos y métodos correlacionados (CFA-CTCM). En el primero, el método no se modela como una variable latente, sino a partir de las correlaciones de las unicidades del mismo método; sin embargo, este procedimiento no es concluyente dado que no es capaz de explicar la varianza que ocasiona el método de medida (Tomás et al., 2013), imposibilitando así que puedan realizarse comparaciones de modelos con y sin el efecto del método en cuanto a la varianza explicada por este. En cambio, en el CFA-CTCM se modela el método mediante la creación de una variable latente, y se puede explicar la varianza atribuible a esta, dado que la varianza de las variables observables se describe como la combinación lineal de los rasgos que se pretenden estudiar (el método y el error). Aparentemente, este procedimiento es el más utilizado en la literatura SEM para evaluar la magnitud de sesgo del método debido a que tiene mayores propiedades descriptivas y eficientes en estudios de simulación (Oliver et al., 2012).

Hasta la fecha, aparentemente no se ha examinado si la varianza del método debido a la existencia de ítems invertidos dentro del IDER impacta sobre la variabilidad de las respuestas; por lo tanto, hay un vacío de conocimientos que puede ser de referencia. Considerando lo anterior, el objetivo del presente trabajo fue evaluar el posible efecto del método asociado a la formulación de ítems invertidos que conforman la subescala eutimia del Inventario de Depresión Estado-Rasgo. Este trabajo se justifica en tanto: (1) brindará una aproximación estructural al estudio del Inventario de Depresión EstadoRasgo en población universitaria, complementaria a la presentada previamente (Dominguez, 2011; Sotelo et al., 2012) y con posible impacto en otros grupos similares; (2) investiga una característica del sesgo potencialmente presente en instrumentos 
de autorreporte construidos con ítems positivos e invertidos, es decir, el sesgo del método; y (3) expondrá evidencia que permita una adecuada interpretación del instrumento estudiado.

\section{Método}

\section{Participantes}

La muestra de estudio estuvo conformada por 357 estudiantes universitarios de la carrera de psicología de una universidad nacional y otra privada ubicadas en Lima Metropolitana (Perú), 147 fueron varones y 210 mujeres, cuyas edades estuvieron entre 16 y 44 años $(M=20,21 ; D E=3,344)$. El género de los participantes fue similar respecto a la edad $(t(355)=0,569 ; p>0,05 ; d=0,006)$ y la distribución de los sujetos en ambas universidades fue aproximadamente del $50 \%$ en cada una. El muestreo utilizado fue de tipo intencional y según el acceso de los autores a las universidades participantes. Todos los sujetos que estuvieron presentes en el momento de administración del instrumento, $\mathrm{y}$ que firmaron un formulario de consentimiento informado, fueron incluidos en la muestra; fueron exceptuados de participar aquellos cuya observación permitió identificarlos con pobre disponibilidad para participar o con posibles patrones de sesgo de respuesta (por respuesta aquiescente).

\section{Instrumento}

Inventario de Depresión Estado-Rasgo (IDER) (Spielberger et al., 2008). Se usó la versión adaptada de Sotelo et al. (2012). Consta de dos secciones de diez ítems en formato tipo Likert cada una, siendo los anclajes respectivos los siguientes: Nada (1), Algo (2), Bastante (3) y Mucho (4) para estado; y Casi nunca (1), Algunas veces (2), A menudo (3) y Casi siempre (4) para rasgo. Dichas subescalas tienen como objetivo principal la evaluación del grado de afectación concurrente (estado) y la frecuencia de ocurrencia (rasgo) del componente afectivo de la depresión, considerando para ello cuatro áreas: distimia y eutimia estado (de cinco ítems cada una), que evalúan el grado de afectación negativa y positiva (respectivamente) presentes en el momento de la evaluación; y distimia y eutimia rasgo (cinco ítems cada una), que se enfocan en la frecuencia de la ausencia de afectividad negativa y positiva (respectivamente). La validez estructural y la confiabilidad ha sido satisfactoriamente corroborada en Perú para la población adulta y adolescente (Dominguez, 2011; Merino et al., 2013; Sotelo et al., 2012).

\section{Procedimiento}

El inventario se administró como parte de un conjunto de otros instrumentos sobre diferentes atributos psicológicos dentro de la institución, lo cual tuvo como finalidad otros objetivos de investigación. El proceso de aplicación de pruebas duró alrededor de tres semanas en las aulas de clase de los estudiantes y en horario regular. Los responsables del estudio explicaron la condición de participación voluntaria, las instrucciones estandarizadas que figuran en el instrumento, monitorearon la participación efectiva y proporcionaron ayuda a las potenciales dudas de los evaluados. Para los análisis que se describen a continuación, primero, se invirtieron las respuestas de los ítems de la sección de eutimia, tanto estado como rasgo, para evitar presentar signos negativos en las cargas factoriales.

Respecto al análisis, se aplicó el procedimiento CFA-CTCM realizado con el programa EQS 6.2 (Bentler \& Wu, 2012). Se utilizó el método de máxima verosimilitud (MV), ya que, aun con indicadores asimétricamente distribuidos, ha mostrado ser adecuado para obtener cargas factoriales (Beaducel \& Herzberg, 2006). La prueba general de bondad de ajuste $\chi^{2}$ fue ajustada por un procedimiento robusto para controlar el efecto de la falta de normalidad de las variables (Satorra \& Bentler, 1994, $\left.S B-\chi^{2}\right)$. Se usaron varios indicadores de 
ajuste más frecuentes en investigación, debido a que ninguno de ellos per se aporta toda la información necesaria para valorar un modelo (Dominguez, 2014; Ferrando \& Anguiano-Carrasco, 2010; Hair, Anderson, Tatham \& Black, 2005; Manzano \& Zamora, 2010; Ruiz, Pardo \& San Martín, 2010; Sánchez \& Sánchez, 1998; Schreiber, Stage, King, Nora \& Barlow, 2006). Estos fueron: el Índice de Aproximación de la Raíz de Cuadrados Medios del Error (RMSEA $\leq$ 0,05; Steiger \& Lind, 1980), el Índice de Ajuste Comparativo ( $\mathrm{CFI} \geq 0,95$; Bentler, 1990) e índices basados en criterios de información Akaike's Information Criterion (AIC; Akaike, 1987) y Consistent Akaike's Information Criterion (CAIC; Bozdogan, 1987). Estos últimos permiten comparaciones entre modelos no anidados.

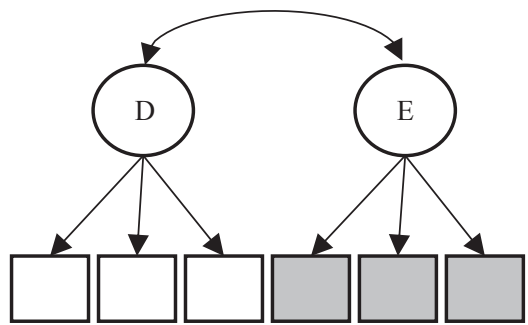

Modelo 1: factores oblicuos, independientemente estimados

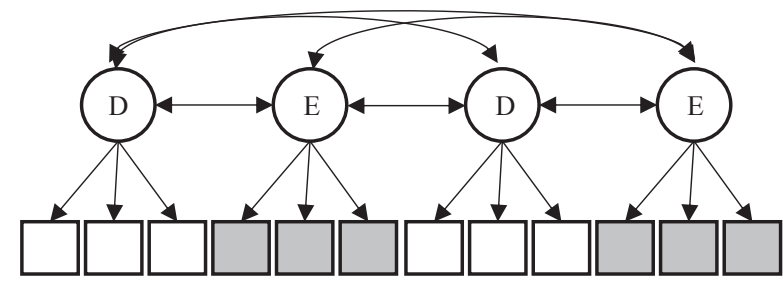

Modelo 3: factores oblicuos, conjuntamente estimados

En gris: ítems de puntuación inversa.

De y Ee: distimia y eutimia estado.

Dr y Er: distimia y eutimia rasgo.

M: factor del método.

IDER: todos los ítems de distimia o eutimia en rasgo o estado.
Los análisis se realizaron sobre la matriz de correlaciones policóricas (Lee, Poon \& Bentler, 1995) debido a que permite estimar la covariación entre las variables continuas subyacentes a los ítems ordinales (Bentler, 2010; Holgado-Tello, Chacón-Moscoso, Barbero-García \& Vila-Abad, 2010), y atenuar el efecto del número de opciones $(<$ cinco) del IDER (Remthulla, Brosseau-Liard \& Savalei, 2012), la esperable falta de normalidad estadística de sus ítems (Lei \& Wu, 2012) y el número de frecuencias cero entre pares de ítems (Lei \& Wu, 2012; Savalei, 2011). Estos aspectos son característicos de los ítems del IDER pues miden un atributo disfuncional en la población esencialmente normal.

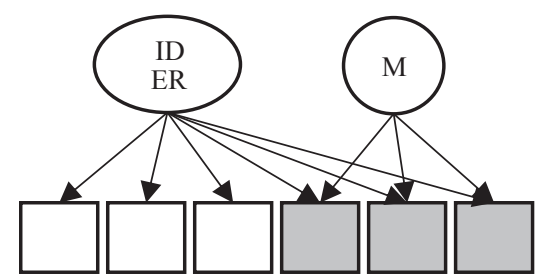

Modelo 2: unidimensional y factor de método $(\mathrm{M})$

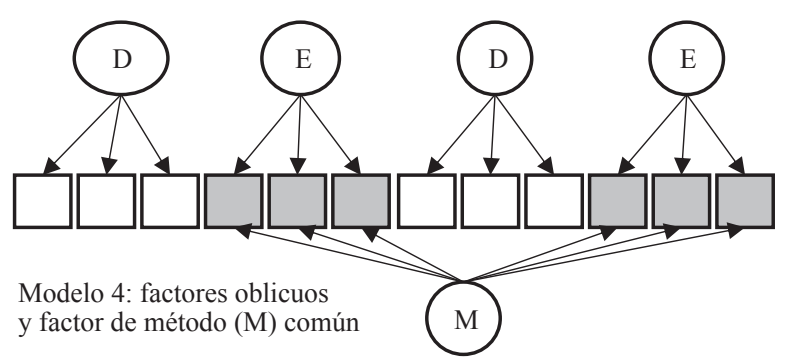

Figura 1. Representación de los modelos evaluados 
En el proceso de modelamiento, se presentaron cuatro modelos para probar su ajuste a los datos: dos de ellos en cada sección, estado y rasgo, y dos modelos con ambas subescalas de forma simultánea. Fue evaluado un primer modelo (M1) de dos factores correlacionados, eutimia y distimia; luego de ello, un segundo modelo (M2) que agrupa todos los ítems en un solo factor, con un factor de método independiente asociado a los ítems del factor eutimia (ítems invertidos). El tercer modelo (M3) evalúa el ajuste de las secciones rasgo y estado simultáneamente, manteniendo el modelo de dos factores oblicuos; esto servirá como línea base para comparar el cuarto modelo (M4), que incluye el factor del método común a las secciones estado y rasgo. Como se verá en los resultados de este análisis específico, los factores del método covarían sustancialmente y pueden formar un solo factor de método común a ambas secciones. La figura 1 resume los modelos evaluados.

\section{Resultados}

\section{Análisis preliminar}

En el análisis preliminar de la asimetría y la curtosis de cada ítem (tabla 1) de la sección estado y rasgo, se observó que la mayor parte de los ítems presentaron valores entre $+1,5$ y $-1,5$, lo cual es considerado o adecuado por la literatura (Pérez \& Medrano, 2010; Ferrando \& Anguiano-Carrasco, 2010); sin embargo, algunos excedían largamente dicho rango, lo que presentaba la situación de aplicar ajustes para atenuar este efecto. La variabilidad de los ítems en sus propias escalas es similar entre ellas y puede considerarse aceptablemente homogénea. Por otro lado, la normalidad multivariada en los presentes datos se evaluó con el coeficiente de Mardia (1970), el cual fue $101,10(Z=61,65)$ y $29,78(Z=18,16)$ para la sección estado y rasgo, respectivamente. Aunque

Tabla 1

Estadísticos descriptivos y correlaciones policóricas IDER

\begin{tabular}{|c|c|c|c|c|c|c|c|c|c|c|}
\hline & IDER 1 & IDER2 & IDER3 & IDER4 & IDER5 & IDER6 & IDER 7 & IDER8 & IDER9 & IDER 10 \\
\hline \multicolumn{11}{|l|}{ Estado } \\
\hline IDER 1 & 1,00 & & & & & & & & & \\
\hline IDER2 & ,82 & 1,00 & & & & & & & & \\
\hline IDER3 &, 58 & ,62 & 1,00 & & & & & & & \\
\hline IDER4 & ,62 & ,63 &, 73 & 1,00 & & & & & & \\
\hline IDER5 & ,64 & ,66 & ,75 & ,64 & 1,00 & & & & & \\
\hline IDER6 &, 33 & ,19 &, 25 &, 24 & ,26 & 1,00 & & & & \\
\hline IDER7 &, 38 & ,40 & ,48 &, 53 & ,43 & ,58 & 1,00 & & & \\
\hline IDER 8 & ,29 &, 28 & ,40 & ,31 &, 30 & ,64 & ,64 & 1,00 & & \\
\hline IDER9 & ,28 &, 30 & ,40 & ,39 &, 35 &, 56 &, 59 &, 77 & 1,00 & \\
\hline IDER 10 &, 23 & ,26 & ,38 &, 37 & ,44 &, 55 & ,47 & ,58 & ,64 & 1,00 \\
\hline $\mathrm{M}$ & 2,64 & 1,35 & 1,39 & 2,54 & 1,17 & 1,23 & 2,96 & 1,36 & 2,75 & 2,83 \\
\hline $\mathrm{DE}$ & ,76 & ,60 & ,65 &, 80 & ,47 &, 51 &, 77 & ,61 &, 80 & ,80 \\
\hline $\mathrm{g}_{1}$ & ,32 & 1,82 & 1,74 & ,23 & 3,22 & 2,59 &,- 26 & 1,84 & ,02 &,- 10 \\
\hline $\mathrm{g}_{2}$ &,- 63 & 3,66 & 2,88 &,- 52 & 4,41 & 3,90 &,- 49 & 3,82 &,- 72 &,- 67 \\
\hline
\end{tabular}




\begin{tabular}{|c|c|c|c|c|c|c|c|c|c|c|}
\hline & IDER11 & IDER 12 & IDER 13 & IDER 14 & IDER 15 & IDER16 & IDER 17 & IDER 18 & IDER 19 & IDER20 \\
\hline \multicolumn{11}{|l|}{ Rasgo } \\
\hline IDER11 & 1,00 & & & & & & & & & \\
\hline IDER 12 & ,61 & 1,00 & & & & & & & & \\
\hline IDER13 &, 53 &, 70 & 1,00 & & & & & & & \\
\hline IDER 14 & ,40 & ,48 &, 54 & 1,00 & & & & & & \\
\hline IDER 15 &, 47 &, 55 &, 49 &, 55 & 1,00 & & & & & \\
\hline IDER 16 &, 34 &, 27 &, 40 &, 33 &, 27 & 1,00 & & & & \\
\hline IDER 17 &, 35 &, 33 &, 35 & ,26 &, 30 & ,60 & 1,00 & & & \\
\hline IDER 18 &, 37 &, 32 & ,31 & ,40 &, 40 &, 51 &, 58 & 1,00 & & \\
\hline IDER 19 &, 43 &, 31 & ,38 &, 27 &, 28 &, 54 &, 72 &, 58 & 1,00 & \\
\hline IDER 20 &, 45 &, 42 & ,33 & ,41 &, 25 &, 56 & ,69 &, 71 & ,66 & 1,00 \\
\hline M & 2,92 & 1,72 & 3,22 & 3 & 3,48 & 1,53 & 1,37 & 1,55 & 1,23 & 2,69 \\
\hline $\mathrm{DE}$ &, 83 & ,63 &, 74 &, 73 &, 67 &, 57 &, 58 &, 59 & ,47 & ,79 \\
\hline $\mathrm{g}_{1}$ &,- 26 & ,64 &,- 55 &,- 17 &,- 05 & ,62 & 1,57 &, 70 & 2,05 &, 01 \\
\hline $\mathrm{g}_{2}$ &,- 70 & 1,02 &,- 47 &,- 67 & ,38 &,- 08 & 1,71 & ,46 & 2,53 &,- 56 \\
\hline
\end{tabular}

$g_{1}:$ asimetría; $g_{2}:$ curtosis.

este último puede considerarse adecuado $(<70$; Rodríguez, \& Ruiz, 2008) y no pondría seriamente en riesgo las estimaciones basadas en el método de máxima verosimilitud en el análisis de ecuaciones estructurales siguiente, la escala anterior se desvía bastante del presupuesto de normalidad multivariada, lo que fortalece la decisión de usar la corrección SB- $\chi^{2}$. Las correlaciones policóricas también muestran buena validez convergente entre los ítems de una misma escala, mientras que se respalda también la validez divergente entre las correlaciones interítems de diferente escala, que son relativamente menores.

\section{Análisis factorial confirmatorio}

Primero, se analizó el impacto del efecto del método independientemente en cada sección del IDER (estado y rasgo), teniendo como presunción: (1) la relativa independencia de la aplicación de ambas secciones en la práctica profesional y de investigación, y (2) que un puntaje único puede ser el uso habitual y parsimonioso del IDER. En el análisis de la sección estado del IDER (tabla 2), se constata que el M1 de dos factores correlacionados, eutimia y distimia, y el M2 que incorpora un factor de método presentan indicadores de ajuste similares. Los RMSEA del M1 y M2 logran intervalos de confianza menores a 0,08 (Hu \& Bentler, $1995)$ y presentan ajustes similares respecto al CFI $(\Delta \mathrm{CFI}=0,003)$. Por otro lado, en lo que respecta a la sección rasgo del IDER (tabla 3), se obtuvieron resultados similares, pues el modelo que incorpora el efecto del método M2 presenta un mejor ajuste, aunque sin ser sustancialmente superior al M1.

Si bien los índices de ajuste son similares en ambos modelos, se observaron cambios relativamente sustanciales a partir de la inclusión del factor del método: la varianza explicada (average variance extracted [AVE]) por los factores disminuyó alrededor de 0,30 y las cargas factoriales de la escala eutimia bajaron entre 0,20 y 0,30 en la subescala de rasgo y entre 0,30 y 0,40 en la de estado. 
Tabla 2

Ajuste de los modelos puestos a prueba en depresión estado

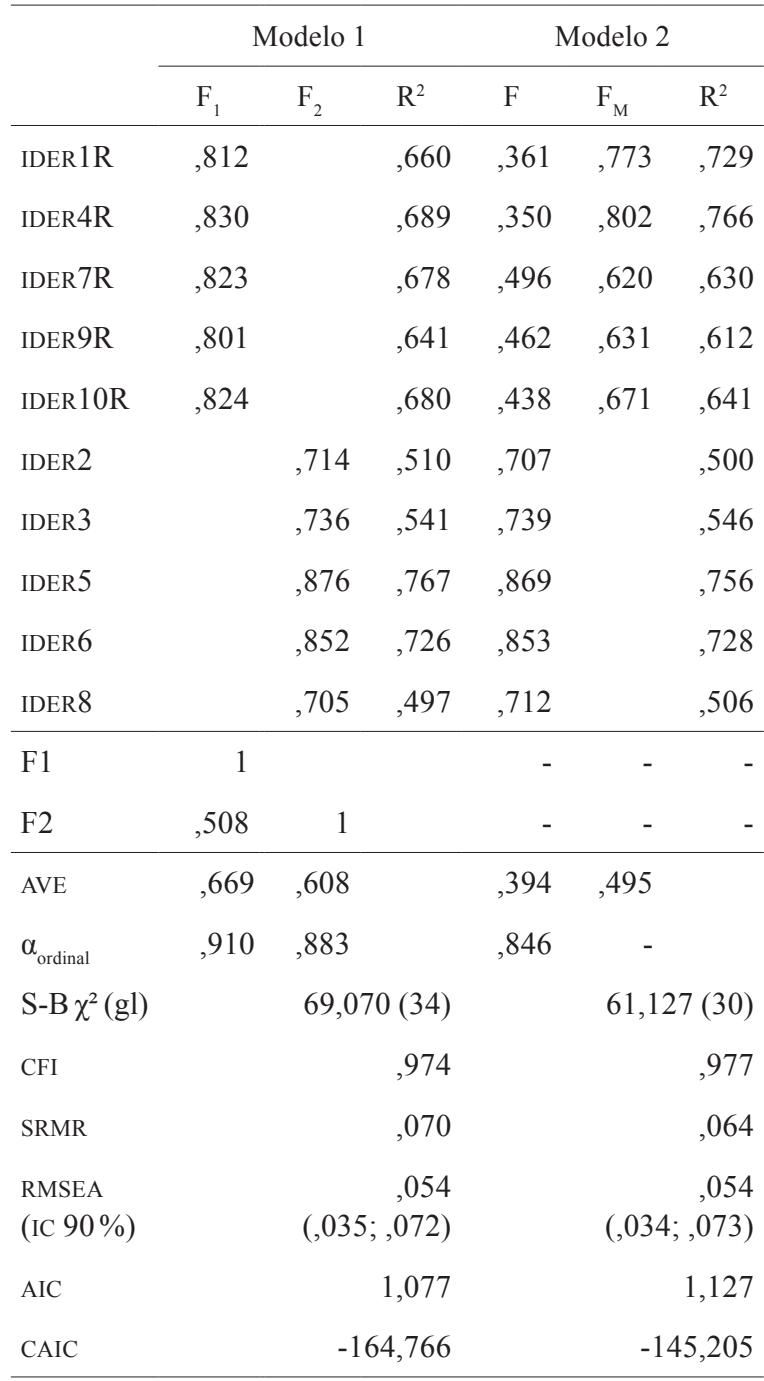

Nota: $\mathrm{R}$ hace referencia a los ítems invertidos. Modelo 1: un factor de eutimia (F1) y un factor de distimia (F2); modelo 2: un factor de depresión global + un factor de método.

En los M3 y M4, se modelaron los ítems de estado y rasgo conjuntamente (tabla 4), cada uno en sus respectivos factores y con las especificaciones iniciales; aquí los modelos de dos factores oblicuos fueron los que mejor se ajustaron en el análisis anterior. Se halló que el ajuste del M3 y M4 fue satisfactorio, además de que las cargas factoriales continuaron siendo fuertes. En el M4, la inclusión del factor del método mejoró claramente los índices de ajuste, pero se observa que no produjo
Tabla 3

Ajuste de los modelos puestos a prueba en depresión rasgo

\begin{tabular}{|c|c|c|c|c|c|c|}
\hline & \multicolumn{3}{|c|}{ Modelo 1 (M1) } & \multicolumn{3}{|c|}{ Modelo 2 (2) } \\
\hline & $\mathrm{F}_{1}$ & $\mathrm{~F}_{2}$ & $\mathrm{R}^{2}$ & $\mathrm{~F}$ & $\mathrm{~F}_{\mathrm{M}}$ & $R^{2}$ \\
\hline IDER11R & ,703 & & ,495 & ,496 & ,502 & ,498 \\
\hline IDER13R &, 835 & & ,697 &, 427 &, 741 &, 731 \\
\hline IDER14R &, 800 & & ,640 & ,434 & 677 & ,647 \\
\hline IDER15R & ,648 & & ,420 &, 420 & ,482 & ,409 \\
\hline IDER20R & ,666 & & ,443 &, 372 & ,543 & ,433 \\
\hline IDER 12 & & 683 & ,466 & 683 & & ,466 \\
\hline IDER 16 & &, 825 & ,680 &, 820 & & ,672 \\
\hline IDER17 & & ,764 & ,583 & ,766 & & ,587 \\
\hline IDER 18 & & ,799 & ,638 & ,799 & & ,639 \\
\hline IDER 19 & &, 851 & ,725 &, 853 & &, 728 \\
\hline F1 & 1 & & & - & - & - \\
\hline $\mathrm{F} 2$ &, 569 & 1 & & - & - & - \\
\hline AVE &, 539 & ,619 & & ,402 & ,357 & \\
\hline$\alpha_{\text {ordinal }}$ &, 850 & ,888 & &, 852 & & \\
\hline $\mathrm{SB}-\chi^{2}(\mathrm{gl})$ & & 61,5 & $4(34)$ & & 53,43 & $2(30)$ \\
\hline CFI & & & ,979 & & & ,982 \\
\hline SRMR & & &, 052 & & &, 044 \\
\hline $\begin{array}{l}\text { RMSEA } \\
\text { (IC } 90 \% \text { ) }\end{array}$ & & $(, 028$ & $\begin{array}{l}, 048 \\
, 066)\end{array}$ & & $(, 025$ & $\begin{array}{l}, 047 \\
, 067)\end{array}$ \\
\hline $\mathrm{AIC}$ & & & 6,406 & & & 6,568 \\
\hline CAIC & & & 2,249 & & & 2,900 \\
\hline
\end{tabular}

Nota: R hace referencia a los ítems invertidos. Modelo 1: un factor de eutimia (F1) y un factor de distimia (F2); modelo 2: un factor de depresión global + un factor de método.

importantes cambios en la varianza explicada (AVE) de los factores, ni en la matriz de covariaciones interfactoriales. El patrón de correlaciones convergentes y divergentes entre las escalas de eutimia y distimia no varió sustancialmente luego de modelar el efecto del método. La varianza del factor del método mostró ser trivial comparada con los factores sustanciales de eutimia (F1 y F3) y distimia (F2 y F4), lo que sugiere su bajo impacto sobre los ítems del IDER. El efecto del factor del método 


\section{Sergio Dominguez-Lara, César merino-Soto}

Tabla 4

Ajuste de los modelos puestos a prueba en depresión rasgo

\begin{tabular}{|c|c|c|c|c|c|c|c|c|c|c|c|}
\hline & \multicolumn{5}{|c|}{ Modelo 3 (M3) } & \multicolumn{6}{|c|}{ Modelo 4 (M4) } \\
\hline & \multicolumn{2}{|c|}{ Estado } & \multicolumn{2}{|c|}{ Rasgo } & \multirow{2}{*}{$\mathrm{R}^{2}$} & \multicolumn{2}{|c|}{ Estado } & \multicolumn{2}{|c|}{ Rasgo } & \multirow{2}{*}{$\mathrm{F}_{\mathrm{M}}$} & \multirow{2}{*}{$\mathrm{R}^{2}$} \\
\hline & $\mathrm{F} 1$ & F2 & F3 & F4 & & $\mathrm{F} 1$ & F2 & F3 & F4 & & \\
\hline I1R &, 808 & & & & ,653 &, 864 & & & &,- 255 &, 811 \\
\hline I4R &, 826 & & & & ,683 &, 861 & & & &,- 200 &, 782 \\
\hline I7R &, 814 & & & & ,663 &, 773 & & & & ,183 & ,632 \\
\hline $\mathrm{I} 9 \mathrm{R}$ & ,802 & & & & ,643 &, 823 & & & &, 437 & ,868 \\
\hline I10R &, 815 & & & & ,664 &, 775 & & & & ,029 &, 601 \\
\hline $\mathrm{I} 2$ & &, 717 & & &, 515 & &, 718 & & & &, 516 \\
\hline $\mathrm{I} 3$ & &, 734 & & &, 538 & &, 733 & & & &, 537 \\
\hline $\mathrm{I} 5$ & & ,908 & & &, 824 & & ,908 & & & &, 825 \\
\hline I6 & &, 847 & & &, 718 & &, 847 & & & &, 718 \\
\hline I8 & &, 710 & & &, 504 & & ,709 & & & &, 502 \\
\hline I11R & & &, 716 & &, 513 & & & ,766 & &,- 160 & ,613 \\
\hline I13R & & & ,807 & & ,652 & & & ,785 & & ,153 & ,640 \\
\hline I14R & & & ,786 & & ,618 & & &, 767 & & ,370 &, 725 \\
\hline $\mathrm{I} 15 \mathrm{R}$ & & & ,638 & & ,407 & & &, 615 & &, 141 & ,398 \\
\hline $\mathrm{I} 20 \mathrm{R}$ & & &, 680 & & ,463 & & & ,666 & &, 027 & ,444 \\
\hline $\mathrm{I} 12$ & & & & 653 &, 427 & & & &, 653 & & ,426 \\
\hline I16 & & & & ,787 &, 620 & & & &, 786 & &, 618 \\
\hline $\mathrm{I} 17$ & & & & ,796 & ,634 & & & & ,797 & & ,635 \\
\hline $\mathrm{I} 18$ & & & & ,752 & ,566 & & & &, 752 & & ,566 \\
\hline I19 & & & & 860 &, 740 & & & &, 861 & &, 741 \\
\hline $\mathrm{F} 1$ & 1 & & & & & 1 & & & & & \\
\hline $\mathrm{F} 2$ &, 478 & 1 & & & &, 457 & 1 & & & & \\
\hline $\mathrm{F} 3$ &, 748 &, 540 & 1 & & &, 741 &, 525 & 1 & & & \\
\hline $\mathrm{F} 4$ & ,453 &, 826 &, 583 & 1 & &, 453 &, 826 &, 589 & 1 & & \\
\hline AVE & ,66 &, 62 &, 53 &, 59 & &, 67 & ,61 &, 52 &, 59 &, 053 & \\
\hline$\alpha_{\text {ordinal }}$ & 907 &, 887 &, 847 &, 878 & & ,911 &, 887 &, 843 &, 879 & & \\
\hline $\mathrm{SB}-\chi^{2}(\mathrm{gl})$ & & & 265,0 & (164) & & & & & 233,6 & $(154)$ & \\
\hline $\mathrm{CFI}$ & & & & ,987 & & & & & & ,990 & \\
\hline SRMR & & & &, 062 & & & & & &, 060 & \\
\hline $\begin{array}{l}\text { RMSEA } \\
\text { (IC 90\%) }\end{array}$ & & & $(, 03$ & $\begin{array}{r}, 042 \\
, 051)\end{array}$ & & & & & $(, 02$ & $\begin{array}{r}, 038 \\
;, 048)\end{array}$ & \\
\hline AIC & & & & 2,982 & & & & & & 74,328 & \\
\hline CAIC & & & & 2,931 & & & & & & 25,499 & \\
\hline
\end{tabular}

Nota: R hace referencia a los ítems invertidos. Modelo 3: cuatro factores correlacionados; modelo 4: cuatro factores correlacionados + un factor de método; F1 y F3: eutimia; F2 y F4: distimia. 
sobre los ítems fue incrementar su carga factorial y en otros ítems, disminuirlo, pero estos cambios fueron pequeños, y las cargas factoriales, luego de remover los efectos del método, continuaron siendo $>0,70$, y, en algunas excepciones, $>0,60$.

\section{Confiabilidad}

Se estimó la confiabilidad de los factores eutimia y distimia en secciones rasgo y estado modelados para observar el efecto del factor del método sobre este parámetro. Para ello, se usó un programa ad hoc (Dominguez, 2012) que calcula la confiabilidad $\alpha$ (Cronbach, 1951) basada en correlaciones policóricas bajo el modelo de factores comunes (Elosua \& Zumbo, 2008; Zumbo, Gadermann \& Zeisser, 2007). La solución factorial utilizada para esta estimación proviene de la tabla 4 (modelo 3 y 4), en la que se presenta también el $\alpha$ ordinal. Los coeficientes estimados en estas soluciones factoriales son prácticamente iguales, lo que puede formalmente representarse por el índice de atenuación propuesto por Gadermann, Guhn y Zumbo (2012). El porcentaje de atenuación debido al factor del método ocurrió entre $\pm 1 \% \mathrm{y} \pm 0 \%$, lo cual indica que no existe algún efecto sustancial sobre la confiabilidad.

\section{Discusión}

El objetivo del presente trabajo fue determinar la presencia del efecto del método en la estructura factorial del Inventario de Depresión Estado-Rasgo, no encontrándose evidencia de ello en la sección tanto de estado como de rasgo. Inicialmente, se probó un modelo bidimensional conforme a los planteamientos de los autores del instrumento, quienes enfatizaron dos factores de contenido, eutimia y distimia. En el presente estudio, esta estructura se respaldó por los resultados correspondientes al primer modelo planteado, el cual se ajusta adecuadamente cuando se consideran los dos factores eutimia y distimia correlacionados, conformados por ítems invertidos y en positivo, respectivamente. Por tanto, esta estructura factorial resultante corresponde con la estructura teórica de la prueba, ya que se agrupan los cinco ítems correspondientes a eutimia y los últimos cinco que conforman la distimia en un segundo factor, en ambas secciones, estado y rasgo.

Como se sustentó inicialmente, quizá dicha distinción se deba más al método empleado (invertir ítems) que a una distinción sustancial de los constructos, de tal manera que uno de los factores deba ser interpretado como el efecto de la manera en que están orientados los ítems. Esa fue la hipótesis de investigación, y con base en esa posibilidad se implementó un segundo modelo en el que, además de un factor general que agrupaba todos los ítems en un solo factor de depresión, se incluyó un factor de método que incorporaba todos los ítems invertidos. Este segundo modelo presentó el mejor ajuste entre los tres modelos propuestos, aunque no se demostró de modo fehaciente la presencia de un factor de método asociado a los ítems invertidos de la escala. Este hallazgo se ve avalado por los índices de ajuste de los modelos de dos factores oblicuos (M1) y el modelo del factor general con un factor de método (M2), ya que ambos presentaban índices de ajuste adecuados y la diferencia entre CFI entre el modelo 2 y 3 fue 0,003 .

En los siguientes análisis se observaron resultados comparativamente diferentes cuando se trabajó con las secciones estado y rasgo conjuntamente, modelando dos factores de método (uno para cada sección). Los resultados indicaron que la influencia de los efectos del método fue casi inexistente, lo que implica que hay evidencias favorables de la solidez de las subescalas eutimia y distimia como variables latentes poco afectadas por este tipo de sesgo. Dichos hallazgos discrepan con estudios previos (Oliver et al., 2012; Tomás, Sancho, Oliver, Galiana \& Meléndez, 2012; Tomás et al., 2013) que indican el efecto del método como un fenómeno ubicuo en instrumentos con ítems invertidos y no invertidos, y que, en consecuencia, se agrupan en 
un factor identificado como el factor de método. Aunque este fenómeno es un problema potencial de los instrumentos de autorreporte, nuestros resultados indican que en el IDER su efecto puede ser considerado trivial. Es posible que la percepción del afecto asociado con la depresión, enmarcado en una clara distinción entre la experiencia actual (estado) y la permanente (rasgo), contribuya a disminuir el efecto del método. Otros aspectos, como las características de la muestra (universitarios) y el proceso de administración del instrumento, pueden aportar varianza única que contribuya a explicar el reducido efecto del método en el IDER. Alguna evidencia reportada sobre la complejidad cognitiva y el nivel socioeconómico de los participantes señala que los patrones de respuesta relacionados con el sesgo son influenciados por la interacción de las características cognitivas, el nivel de instrucción y socioeconómico (Bachman \& O’Malley, 1984; Baron-Epel, Kaplan, Weinstein $\&$ Green, 2010). Aunque los presentes hallazgos arrojaron que el efecto del sesgo es trivial en la muestra de universitarios, no se investigaron otras fuentes de sesgo plausibles, como la respuesta extrema o aquiescencia, por lo tanto, los resultados pueden ser de referencia para el tipo de sesgo investigado.

En cuanto a las implicancias de los hallazgos, queda claro, al menos en esta muestra, que la interpretación de los resultados de las secciones estado y rasgo puede realizarse válidamente en términos de eutimia y distimia, ya que ambos factores representan facetas distintas del estado de ánimo, desde el punto de vista conceptual y empírico. En segundo lugar, el IDER parece ser un instrumento robusto pues sus puntajes no se atenúan seriamente por el efecto del método en la muestra de universitarios de una población normal esencialmente no clínica. Tercero, y como corolario de lo anterior, el efecto del método fue insustancial para la consistencia interna, la cual es alta para los puntajes de eutimia y distimia, ya que, al ser cercana a 0,90 , el uso de estos puntajes puede satisfacer los niveles conservadores que se requieren en la práctica clínica diagnóstica (Nunnally \& Bersntein, 1995).

Entre las limitaciones del presente estudio cabe destacar las características de la muestra, la cual estuvo conformada solo por estudiantes universitarios y de cierto rango de edad; por lo tanto, la replicación de este estudio en población clínica o general no universitaria es una cuestión abierta a verificación, considerando que el efecto del método puede variar según la muestra o el contenido del instrumento (Tomás et al., 2012). Por otro lado, y como consecuencia de lo anterior, los efectos potenciales de la diferencia de género y el nivel de instrucción no fueron incluidos en los análisis, y se requiere tomarlos en cuenta como posibles variables moderadoras. Este estudio concluye que el IDER es poco afectado por la presencia de ítems en sentido opuesto al estado de ánimo depresivo (eutimia), tanto en su validez interna como en las estimaciones de consistencia interna, $\mathrm{y}$, por lo tanto, la variabilidad de las respuestas se debe esencialmente a los constructos subyacentes y medidos por el IDER.

\section{Referencias}

Agudelo, D. (2009). Propiedades psicométricas del Inventario de Depresión Estado/Rasgo (IDER) con adolescentes y universitarios de la ciudad de Bucaramanga. Pensamiento Psicológico, 5(12), 139-160.

Agudelo, D. Spielberger, C. \& Buela-Casal, G. (2007). La depresión: ¿un trastorno dimensional o categorial? Salud Mental, 30, 20-28.

Agudelo, D., Carretero-Dios, H., Blanco Picabia, A., Pitti, C., Spielberger, C. \& Buela- Casal, G. (2005). Evaluación del componente afectivo de la depresión: análisis factorial del ST/DEP revisado. Salud Mental, 28, 32-41.

Agudelo, D., Gómez, Y. \& López, P. (2014). Propiedades psicométricas del Inventario de Depresión Estado Rasgo (IDER) con una muestra 
de población general colombiana. Avances en Psicología Latinoamericana, 32(1), 71-84.

Akaike, H. (1987). Factor analysis and AIC. Psychometrika, 52, 317-332.

Bachman, J. G. \& O’Malley, P. M. (1984). Yea-saying, nay-saying and going to extremes: Black-White differences in response styles. Public Opinion Quarterly, 48, 491-509.

Baron-Epel, O., Kaplan, G., Weinstein, R. \& Green, M. S. (2010). Extreme and acquiescence bias in a bi-ethnic population. European Journal of Public Health, 20(5), 543-8

Beaducel, A. \& Herberg, P. Y. (2006). On the performance of maximum likelihood versus means and variance adjusted weighted least squares estimation in CFA. Structural Equation Modeling: A Multidisciplinary Journal, 13(29), 186-203.

Beck, A., Steer, R. \& Brown, G. (2006). Inventario de Depresión de Beck. Buenos Aires: Paidós.

Bentler, P. M. (1990). Comparative fit indexes in structural models. Psychological Bulletin, 107, 238-246.

Bentler, P. M. (2010). SEM with simplicity and accuracy. Journal of Consumer Psychology, 20, 215-220.

Bentler, P. M. \& Wu, E. J. C. (2012). EQS 6.2 for windows [Statistical Program]. Encino: Multivariate Software, Inc.

Bozdogan, H. (1987). Model selection and Akaike's information criteria (AIC): the general theory and its analytical extensions. Psychometrika, 52, 345-370.

Campbell, D. \& Fiske, D. (1959). Convergent and discriminant validation by the multitrait-multimethod matrix. Psychological Bulletin, 56, 81-105.

Carmines, E. \& Zeller, R. (1979). Reliability and validity assessment. Londres: Sage.

Cronbach, L. J. (1951). Coefficient alpha and the internal structure of tests. Psychometrika, 16, 297-334
Dominguez, S. (noviembre, 2011). Propiedades Psicométricas del Inventario de Depresión Estado Rasgo en estudiantes universitarios. Ponencia presentada en el VIII Congreso Nacional de Profesionales y Estudiantes de Psicología, Facultad de Psicología y Trabajo Social, Universidad Inca Garcilaso de la Vega, 2 al 4 de noviembre, Lima, Perú.

Dominguez, S. (2012). Propuesta para el cálculo del alfa ordinal y theta de Armor. Revista de Investigación en Psicología, 15, 213-217.

Dominguez, S. (2014). Análisis Psicométrico de la Escala de Bienestar Psicológico para Adultos en estudiantes universitarios de Lima: un enfoque de ecuaciones estructurales. Psychologia: Avances en la Disciplina, 8(1), 23-31.

Elosua, P. \& Zumbo, B. D. (2008). Coeficientes de fiabilidad para escalas de respuesta categórica ordenada. Psicothema, 20, 896-901.

Ferrando, P. \& Anguiano-Carrasco, C. (2010). El análisis factorial como técnica de investigación en psicología. Papeles del Psicólogo, 31(1), 18-33.

Ferrando, P. \& Lorenzo-Seva, U. (2010). Acquiescence as a source of bias and model person misfit: a theoretical and empirical analysis. British Journal of Mathematical and Statistical Psychology, 63, 427-448.

Gadermann, A. M., Guhn, M. \& Zumbo, B. D. (2012). Estimating ordinal reliability for Likert-type and ordinal item response data: a conceptual, empirical, and practical guide. Practical Assessment, Research and Evaluation, 17(3). Recuperado de http://pareonline.net/pdf/v17n3.pdf

Hair, J., Anderson, R., Tatham, R. \& Black, W. (2005). Análise multivariada de dados. Porto Alegre: Bookman.

Holgado-Tello, F. P., Chacón-Moscoso, S., Barbero-García, I. \& Vila-Abad, E. (2010). Polychoric versus Pearson correlations in exploratory and confirmatory factor analysis of ordinal variables. Quality and Quantity, 44(1), 153-166.

Hu, L. T. \& Bentler, P. M. (1995). Evaluating model fit. En R. H. Hoyle (ed.), Structural equation 
modeling: concepts, issues, and applications (pp. 76-99). Thousand Oaks: Sage.

Krosnick, J. A. (1999). Survey research. Annual Review of Psychology, 50, 537-567.

Lance, C., Dawson, B., Birkelbach, D. \& Hoffman, B. (2010). Method effects, measurement error, and substantive conclusions. Organizational Research Methods, 13(3), 435-455.

Lee, S.-Y., Poon, W.-Y. \& Bentler, P. M. (1995). A two-stage estimation of structural equation models with continuous and polytomous variables. British Journal of Mathematical and Statistical Psychology, 48, 339-358.

Lei, P.-W. \& Wu, Q. (2012). Estimation in structural equation modeling. En R. H. Hoyle (ed.), Handbook of structural equation modeling (pp. 164-179). Nueva York: Guildford Press.

Manzano, A. \& Zamora, S. (2010). Sistema de ecuaciones estructurales: una herramienta de investigación. México D.F.: Centro Nacional de Evaluación para la Educación Superior.

Mardia, K. (1970). Measures of multivariate skewness and kurtosis with applications. Biometrika, 57, 519-530.

Marsh, H. W. (1996). Positive and negative global self-esteem: a substantively meaningful distinction or artifactors? Journal of Personality and Social Psychology, 70(4), 810-819.

Merino, C., Pflucker, D. \& Riaño-Hernández, D. (2013). Análisis factorial exploratorio del Inventario de Depresión Estado-Rasgo (ST-DEP) en adolescentes. Diversitas: Perspectivas en Psicología, 8(2), 319-330.

Nunnally, J. (1978). Psychometric theory (2a ed.). Nueva York: McGraw-Hill.

Nunnally, J. C. \& Bernstein, I. J. (1995). Teoría psicométrica ( $\left.3^{\mathrm{a}} \mathrm{ed}\right)$. México D.F.: McGraw-Hill Latinomericana.

Ocampo, L. (2007). Análisis correlacional del Cuestionario de Depresión Estado/Rasgo (ST/DEP) con una muestra de adolescentes y universitarios de la ciudad de Medellín (Colombia). Psicología desde el Caribe, 20, 28-49.
Oliver, A., Sancho, P., Galiana, L., Tomás, J. \& Gutiérrez, M. (2012). Efecto de método asociado a los ítems en negativo en la versión en portugués de la escala de autoestima de Rosenberg. Revista Iberoamericana de Diagnóstico y Evaluación Psicológica, 33(1), 75-91.

Pérez, E. \& Medrano, L. (2010). Análisis factorial exploratorio: bases conceptuales y metodológicas. Revista Argentina de Ciencias del Comportamiento, 2(1), 58-66.

Podsakoff, P., MacKenzie, S., Lee, J. \& Podsakoff, N. (2003). Common method biases in behavioral research: a critical review of the literature and recommended remedies. Journal of Applied Psychology, 88(5), 879-903.

Remthulla, M., Brosseau-Liard, P. É. \& Savalei, V. (2012). When can categorical variables be treated as continuous? A comparison of robust continuous and categorical SEM estimation methods under suboptimal conditions. Psychological Methods, 17(3), 354-373.

Rodríguez, M. \& Ruiz, M. (2008). Atenuación de la asimetría y de la curtosis de las puntuaciones observadas mediante transformaciones de variables: Incidencia sobre la estructura factorial. Psicológica, 29, 205-227.

Rosenberg, M. (1965). Society and the adolescent child. Princeton: Princeton University Press.

Ruiz, M., Pardo, A. \& San Martín, R. (2010). Modelos de ecuaciones estructurales. Papeles del Psicólogo, 31(1), 34-45.

Sánchez, E. \& Sánchez, M. (1998). Los modelos de estructuras de covarianza como método de validación de constructo. En V. Manzano \& M. Sánchez (comps.), Investigación del comportamiento. Innovaciones metodológicas y estrategias de docencia (pp. 101-112). Sevilla: Instituto Psicosociológico Andaluz de Investigaciones.

Satorra, A. \& Bentler, P. M. (1994). Corrections to test statistics and standard errors in covariance structure analysis. En A. von Eye \& C. C. Clogg (eds.), Latent variables analysis: 
applications for developmental research (pp. 399-419). Thousand Oaks: Sage.

Savalei, V. (2011). What to do about zero frequency cells when estimating polychoric correlations. Structural Equation Modeling: A Multidisciplinary Journal, 18(2), 253-273.

Schaeffer, N. C. \& Presser, S. (2003). The science of asking questions. Annual Review of Psychology, 29, 65-88.

Schreiber, J., Stage, F., King, J., Nora, A. \& Barlow, E. (2006). Reporting structural equation modeling and confirmatory factor analysis results: a review. The Journal of Education Research, 99(6), 323-337.

Sotelo, L., Sotelo, N., Dominguez, S., Cueto, E., Alarcón, D., Poma, I., Padilla, O. \& Barboza, M. (2012). Propiedades psicométricas del Inventario de Depresión Estado-Rasgo (IDER) en una muestra de Adultos de Lima Metropolitana. Avances en Psicología Latinoamericana, 20(2), 59-68.

Spector, P. (2006). Method variance in organizational research. Truth or urban legend? Organizational Research Methods, 9(2), 221-232.

Spielberger, C., Agudelo, D. \& Buela-Casal, G. (2008). Inventario de Depresión Estado/ Rasgo (IDER). Madrid: TEA Ediciones.

Steiger, J. H. \& Lind, J. C. (mayo, 1980). Statistically based tests for the number of common factors. Ponencia presentada en la reunión anual Psychometric Society, Iowa City, IA.

Tomás, J. \& Oliver, A. (1999). Rosenberg's self-esteem scale: two factor or method effects.
Structural Equation Modeling: A multidisciplinary Journal, 6(1), 84-98.

Tomás, J., Galiana, L., Hontangas, P., Oliver, A. \& Sancho, P. (2013). Evidencia acumulada sobre los efectos de método asociados a ítems invertidos. Psicológica, 34, 365-381.

Tomás, J., Sancho, P., Oliver, A., Galiana L. \& Meléndez, J. (2012). Efectos de método asociados a ítems invertidos vs. ítems en negativo. Revista Mexicana de Psicología, 29(2), 105-115.

Vera-Villarroel, P., Buela-Casal, G., Celis-Atenas, K., Córdova-Rubio, N., Encina-Olea, N. \& Spielberger, C. (2008). Chilean experimental version of the State-Trait Depression Questionnaire (ST-DEP): Trait Sub-scale (T-DEP). International Journal of Clinical and Health Psychology, 8(2), 563-575.

World Health Organization (WHO) (2002). World Health Report 2002. Reducing risks, promoting Healthy Life. Ginebra: World Health Organization.

World Health Organization (WHO) (2010). Mental health and development: targeting people with mental health conditions as a vulnerable group. Ginebra: World Health Organization.

Zumbo, B. D., Gadermann, A. M. \& Zeisser, C. (2007). Ordinal versions of coefficients alpha and theta for Likert rating scales. Journal of Modern Applied Statistical Methods, 6, 21-29.

Zung, W. (1965). A self-rating depression scale. Archive General of Psychiatry, 12, 63-70. 
